\title{
ESTUDO DA INCIDÊNCIA DE COLEDOCOLITÍASE EM PACIENTES COM COLECISTITE CALCULOSA AGUDA E CRÔNICA SUBMETIDOS À COLECISTECTOMIA VÍDEOLAPAROSCÓPICA
}

\author{
STUDY OF INCIDENCE OF CHOLEDOCHOLITIASIS: IN PATIENTS WITH ACUTE AND \\ CHRONIC CALCULOUS CHOLECYSTITIS OPERATED BY LAPAROSCOPIC \\ CHOLECISTECTOMY
}

\author{
Sidney Moreno Gil, TCBC-SP' ; Juarez Fortunato Braga, TCBC-SP²; \\ Sérgio Antônio R. Centurion, TCBC-SP²; Bruno Ziade Gil, ACBC-SP³
}

\begin{abstract}
RESUMO: Objetivo: Avaliar a incidência da coledocolitiase em pacientes submetidos à colecistectomia laparoscópica, portadores de colecistopatia calculosa aguda e crônica. O presente estudo, também, analisa se a era laparoscópica modificou a incidência e a história natural da litíase da via biliar quando compara os dados da literatura com os índices do Grupo estudado. Método: O estudo foi realizado em um Grupo de 946 pacientes, distribuídos em Grupo A de 214 pacientes de 1991-1995 e Grupo B de 732 pacientes de 1999-2007 colecistectomizados pelo método laparoscópico. O critério diagnóstico de coledocolitíase foi estabelecido por colangiofluoroscopia de rotina em todas as operações. Resultados: A incidência total de coledocolitiase no Grupo A de $9,8 \%$ e no $\mathrm{B}$ de 5,8\% não mostram diferença estatística significativa entre si e com a literatura mundial $(\mathrm{p}=0,08)$. Nos pacientes operados por colecistite aguda também não se observou diferença estatística entre o grupo A e B na incidência de litíase da via biliar( $p=0,8)$. A análise dos dados nos pacientes operados por colecistite crônica revela uma taxa de coledocolitíase menor no Grupo B de 3,7\% com significância estatística do que no A de $8,4 \%$ p=0,03. Conclusão: $\mathrm{O}$ presente estudo revela uma diminuição expressiva na incidência de coledocolitiase no Grupo B em relação ao A e a literatura mundial quando a indicação cirúrgica ocorre na fase não complicada da doença litiásica biliar. $O$ estudo também demonstra um aumento significativo na indicação da colecistectomia laparoscópica eletiva mais precoce no Grupo B como já observado em diversas publicações da literatura médica (Rev. Col. Bras. Cir. 2007; 34(4): 214-217).
\end{abstract}

Descritores: Coledocolitíase; Colecistectomia Laparoscópica; Icterícia.

\section{INTRODUÇÃO}

A história natural da coledocolitiase é diversa e geralmente imprevisível. A apresentação clínica dos cálculos de coledoco pode ocorrer basicamente de quatro formas. Os denominados cálculos silenciosos menores podem cursar sem sintomas, muitas vezes migrando espontaneamente para o duodeno. Outras vezes podem produzir obstrução do ducto biliar causando icterícia, e em outras situações, um quadro de colangite. A pancreatite aguda seria a quarta condição clínica na apresentação da coledocolitiase.

A litiase biliar é uma doença de alta prevalência na população geral - $10 \%$ e a incidência da coledocolitiase têm uma frequiência de 8 a $15 \%$.1,2. Dados estatísticos obtidos de várias instituições americanas revelaram 9,3\% de coledocolitiase, num total de 2.982 pacientes operados de colecistite aguda calculosa e de $10.5 \%$ dentre 12428 pacientes operados de colecistite crônica calculosa ${ }^{3}$. Outras publicações como Glenn em um minucioso estudo de 4.677 pacientes operados por colescistite crônica calculosa, informou uma incidência de 8,8\% de coledocolitiase; assim como Bolton revisando publicações de 30 anos, encontrou taxas de 6 a
19,5\% de cálculos da via biliar ${ }^{4}$. Algumas publicações reportam que a incidência atual de coledocolitiase é de 3\% a 6\% , sendo que este declínio provavelmente deve-se à colecistectomia laparoscópica eletiva mais precoce ${ }^{5-6}$. Outros artigos da literatura na era laparoscópica sobre incidência de coledocolitiase referem taxas de $10-11.3 \%$, porém sem especificar se ocorrem diferenças estatísticas entre colecistite crônica e aguda calculosa $a^{7-10}$.

O objetivo do presente estudo é não só avaliar a incidência de coledocolitiase num total de 1152 pacientes submetidos à colecistectomia laparoscópica de 1991 - 2007 no Hospital São Domingos, Catanduva - SP, portadores de colescistopatia calculosa aguda e crônica, como verificar também se o método laparoscópico atualmente, modificou a incidência da litíase da via biliar e, portanto, sua história natural, quando comparados os dados da literatura com os do Grupo estudado.

\section{MÉTODO}

Foram realizados, no período de 1991 a 2007, 1152 colecistectomias laparoscópicas por colecistite calculosa crô-

\footnotetext{
1. Diretor CCIH, Presidente da COREME do Hospital São Domingos - Catanduva - SP.

2. Cirurgião do Departamento de Cirurgia do Hospital São Domingos - Catanduva - SP.

3. Pós-Graduando em Gastrocirurgia do Hospital Real e Benemérita Sociedade Portuguesa de Beneficência - São Paulo.

Recebido em 06/11/2006

Aceito para publicação em 08/01/2007

Conflito de interesses: nenhum

Fonte de financiamento: nenhuma

Trabalho realizado no Departamento de Cirurgia do Hospital São Domingos - Catanduva - SP.
} 
nica e aguda, sendo que para o presente estudo foram incluídos apenas 946 pacientes nos quais o critério diagnóstico de coledocolitiase foi estabelecido pela realização de colangiofluoroscopia operatória de rotina em todas as operações ${ }^{11,12}$. Foram excluídos 206 pacientes operados entre 1995 1998, em cujo Grupo a colangiofluoroscopia operatória foi indicada com critérios de seletividade, assim como oito pacientes operados de colecistite aguda alitiásica.

Os 946 pacientes foram distribuídos em dois Grupos :A) Pacientes operados no período de 1991 - 1995 e B) Pacientes operados entre 1999 - 2007. Todas as operações foram realizadas pela mesma equipe e a colangiofluoroscopia operatória, por uma quinta punção, injetando-se $10 \mathrm{ml}$ de solução iodada como padrão diagnóstico da coledocolitiase ${ }^{11,12}$.

Os pacientes foram classificados de acordo com os dados clínicos, laboratoriais, ultrassonográficos e anatomopatológicos, em portadores de colecistite crônica ou aguda.

Para análise dos resultados, aplicou-se o teste do Qui-quadrado, com o objetivo de estudar a incidência da coledocolitiase nos Grupos A e B, determinando se há diferença estatística entre os mesmos, aceitando-se como valor de significância estatística $\mathrm{p}<0,05$.

\section{RESULTADOS}

Foram encontrados 214 pacientes para o Grupo A sendo 154 (71,9\%) com colecistite crônica e 60 com colecistite aguda; e para o Grupo B 732 pacientes sendo 588 com colecistite crônica e 144 com colecistite aguda (Tabela 1). A distribuição semelhante quanto ao sexo - masculino $28 \%$ e feminino $72 \%$. A idade média foi de 53,4 anos.

A Tabela 2 mostra os resultados da incidência total de coledocolitiase nos Grupos A e B, assim como mostra os resultados segundo o diagnóstico de colecistite aguda e crônica.

A análise dos dados das Tabelas 1 e 2 nos permite afirmar que :

- quando comparamos a incidência total de coledocolitiase no Grupo A - 9,8\% e no Grupo B - 5,8\%, com os dados da literatura, não se observa diferença estatisticamente significante com $\mathrm{p}=0,08$.

- não há diferença estatística significante do número de pacientes operados por colecistite crônica entre o Grupo A e $\mathrm{B}$ com $\mathrm{p}=0,46$.

- O presente estudo revela diminuição com significância estatística de colecistite aguda calculosa operado no grupo B em relação ao Grupo A com p=0,04.

- no Grupo A não há diferença estatística na incidência da coledocolitiase entre os pacientes operados de colecistite crônica $(8,4 \%)$ e colecistite aguda $(13,3 \%)$ $\mathrm{p}=0,3$.

- no Grupo B, observa-se diferença estatística na incidência de coledocolitiase entre colecistite crônica $(3,7 \%)$ e colecistite aguda $(15,5 \%)$ com $p<0,01$.

- não há diferença estatística na incidência de coledocolitiase nos Grupos A e B operados de colecistite aguda com $\mathrm{p}=0,8$.
- quando comparamos os pacientes operados de colecistite crônica observamos diferença estatística significante na incidência de coledocolitiase do Grupo A 8,4\% e Grupo B 3,7\% com $\mathrm{p}=0,03$.

\section{DISCUSSÃO}

A historia natural da colelitiase assintomática tem sido estudada em varias populações e a conduta terapêutica permanece controversa. Alguns artigos citam que os sintomas biliares ocorrem em torno de 2 a $4 \%$ ao ano, resultando em poucas complicações, sendo que um deles teve seguimento por 24 anos. Nesses estudos não há referência da incidência de coledocolitiase ${ }^{13,14-17}$.

O tratamento cirúrgico da colecistite calculosa iniciou-se em 1664, quando Stalpert Von Der Weil mencionou a retirada de cálculos biliares ao drenar um abscesso de parede abdominal evoluindo até a primeira colecistectomia aberta, em 1867, nos EUA, realizada por John Bobbs ${ }^{15}$.

A colecistectomia aberta permaneceu como única opção cirúrgica para doenças da vesícula biliar até 1987, quando Mouret, na França, realizou a primeira operação pelo método laparoscópico, popularizada posteriormente por Dubois e associados $^{5-16}$

A indicação para colecistectomia aberta ocorria geralmente diante de complicações da doença litiásica biliar como colecistite aguda ou icterícia. Com avanço dos métodos diagnósticos, principalmente o ultra-som e a introdução da cirurgia laparoscópica, observou-se um aumento significativo nas indicações da colecistectomia, determinada pelo mesmo nível de segurança da técnica convencional, dor menos intensa no período pós-operatório com retorno precoce às atividades, menor permanência hospitalar e melhor resultado estético. Há relatos que reportam aumento de $28 \%$ nas indicações da colecistectomia laparoscópica ${ }^{17-19}$ e os dados dos grupos estudados mostram que foram operados aproximadamente $75 \%$ a mais de pacientes/ano no grupo B do que no Grupo A.

Tabela 1 - Distribuição de pacientes, de acordo com o diagnóstico, em colecistite crônica e aguda.

\begin{tabular}{lrccc}
\hline Diagnóstico & \multicolumn{2}{c}{ A } & \multicolumn{2}{c}{ B } \\
\cline { 2 - 5 } & $\mathbf{n}$ & $\boldsymbol{\%}$ & $\mathbf{n}$ & $\%$ \\
\hline C. crônica calculosa & 154 & 71,9 & 588 & 80,4 \\
C. aguda calculosa & 60 & 28,1 & 144 & 19,6 \\
Total & 214 & 100 & 732 & 100 \\
\hline
\end{tabular}

Tabela 2 - Incidência total de coledocolitiase nos grupos $A$ e $B$ e segundo o diagnóstico de colecistite crônica e aguda.

\begin{tabular}{lrrrr}
\hline Diagnóstico & \multicolumn{2}{c}{ A } & \multicolumn{2}{c}{ B } \\
\cline { 2 - 5 } & \multicolumn{1}{c}{$\mathbf{n}$} & \multicolumn{1}{c}{$\%$} & n & \multicolumn{1}{c}{$\%$} \\
\hline C. crônica calculosa & 13 & 8,4 & 22 & 3,7 \\
C. aguda calculosa & 8 & 13,3 & 21 & 14,5 \\
Total & 21 & 9,8 & 43 & 5,8 \\
\hline
\end{tabular}


O presente estudo revela o aumento expressivo de colecistectomia no grupo B com indicação eletiva precoce ou mesmo em pacientes assintomáticos, e inversamente foi operado um número significativamente menor de pacientes com colecistite aguda no Grupo B quando comparados ao Grupo A com $\mathrm{p}=0,04$.

A colangiografia operatória introduzida por Mirizzi em 1932:16-18 critério de eleição para diagnóstico da coledocolitiase, revelou que a incidência total no Grupo A de 9,8\% e no Grupo B de 5,8\% não apresenta diferenças estatísticas significativas entre si; assim como também com os dados coletados na literatura médica de 8 a 15\%. Em relação à colecistite aguda calculosa, não ocorreu diferença estatística significativa entre os dois Grupos $(\mathrm{p}=0,8)$. Quando analisamos a incidência de coledocolitiase na colecistite crônica calculosa do Grupo B - 3,7\% e do grupo A - 8,4\% observa-se diferença estatística significativa entre ambos com $\mathrm{p}=0,03$.
O cotejamento dos dados estudados permite-nos afirmar que o método laparoscópico permitiu um aumento expressivo nas indicações cirúrgicas da colecistectomia, sobretudo na fase ainda sem complicações da litíase, reduzindo a taxa de coledocolitiase do Grupo B quando comparada ao Grupo A e às publicações da literatura médica anteriores à videolaparoscopia. Desta forma a operação videolaparoscópica introduz um fator novo que poderá modificar a história natural da coledocolitiase, diminuindo a incidência da mesma e de suas potênciais complicações.

\section{AGRADECIMENTOS}

Ao Dr. Neder José Abdo, chefe da UTI do Hospital São Domingos pelos cálculos estatísticos aplicados para a realização deste trabalho.

\begin{abstract}
Background: To evaluate the incidence of choledocholitiasis in patients submitted to the laparoscopic cholecystectomy, carriers of acute and chronic calculous cholecystitis. The present study also analyses if the laparoscopic age modified the incidence and the natural history of lithiasis of the biliary duct when it compares the data of literature with yhe indices of the studied group. Method: The study was carried out in a group of 946 patients subdivided in group A of 1991 - 1995 and group B of 732 patients of 1999-2007 submitted to the cholecystectomy by the laparoscope method. The diagnostic criterion of choledocholithiasis was estabilished by cholangiofluroscopy of routine in all surgeries. Results: The total incidence of choledocholithiasis in the group $A-9,8 \%$, and in the group B-5,8\% doesn't show difference significant statistics between itself and with the world-wide literature $(p=0,08)$. In the patients operated for acute cholecystitis difference statistics was not also observed between group $A$ and $B$ in the incidence of lithiasis of the biliary duct $(p=0,8)$. The analysis of the data in operated patients for chronic cholecystitis discloses a smaller tax of choledocholitiasis in group B of 3,7\% with significant statistics in the group A of 8,4\% p=0,03. Conclusion: The present study discloses an expressive reduction in the incidence of choledocholitiasis in group $B$ in relation to the A and worl-wide literature when the surgical indication occurs in the not complicated phase of the biliary lithiasic illness. The study demonstrates a significant increase in the indication of precocious elective laparoscopic cholecystectomy in group $B$ already observed in diverse publications of medical literature.
\end{abstract}

Key words: Choledocholithiasis; Laparoscopic surgery; Jaundice.

\section{REFERÊNCIAS}

1. Ortigara L, Enilde E. Guerra EE. Avaliação pré-operetória dos pacientes com coledocolitiase. Rev. Técnico Cientifica do Grupo Hospitalar Conceição 2005. p. 8-16

2. Freitas ML, Bell RL, Duffy AJ. Choledocholithiasis: evolving standards for diagnosis and management. World J Gastroenterol. 2006;12(20):3162-7.

3. Bockus HL. Gastroenteorologia Tomo III - Coledocolitiasis $-2^{\mathrm{a}}$ edicion. - Barcelona; Salvat Editores. 1968. p.826-44.

4. Maingot. Operaciones Abdominales. Coledocolitiasis. $8^{\text {a }}$ Edicion Panamericana Editora 1986. p.1817-1839

5. Marcel AC, Machado J, Silva RM. Tratamento Laparoscópico de Coledocolitiase 2000 Arq.Gastroenterol. 2000;37(3) Available from: www.scielo.br/scielo.php

6. Collins C, Maguire D, Ireland A, Fitzgerald E, O'Sullivan GC. A prospective study of common bile duct calculi in patients undergoing laparoscopic cholecystectomy: natural history of choledocholithiasis revisited. Ann Surg. 2004; 239(1):28-33.

7. Rojas Ortega S, Arizpe Bravo D. Exploration of billiary tracts for laparoscopy for treatment of choledocholithiasis - Rev Gastroenterol Mex. 2004; 69 Suppl 3:112 - 6.
8. Duensing RA, Williams RA. Commmon bile duct stone characteristics: correlation with treatment choice during laparoscopic cholecystectomy. J Gastrointest Surg. 2000; 4(1):6 -12.

9. Petelin JB. Laparoscopic common bile duct exploration. Surg Endosc. 2003; 17(11) :1705-15. Epub 2003 Sep 10.

10. Tazuma S. Gallstone disease: epidemiology, pathogenesis, and classification of biliary stones ( common bile duct and intrahepatic). Best Pract Res Clin Gastroenterol. 2006; 20(6):1075-83.

11. Wenner DE, Whitwam P, Turner D. Actual time required for dynamic fluoroscopic intaoperative cholangiography. JSLS. 2005; 9(2):174-7.

12. Shively EH, Wieman TJ. Operative cholangiography. Am J Surg. 1990; 159(4):380-4; discussion 385.

13. Gracie W, Ransohoff D. The natural history off silent gallstones. The innocent gallstone is not a myth. N Engl J Méd. 1982; 307 : 798.

14. Attili AF, De Santis A, Capri R, Repice AM, Maselli S. The natural history of gallstones: the GREPCO experience. The GREPCO Group. Hepatology. 1995 Mar;21(3):655-60.

15. Robert E, NN Hermann NN. Cirurgia para colecistite aguda e crônica. Clín Cirúr Am N. 1990; 6:1333 -45. 
16. Cueto J, Weber A. Cirurgia laparoscópica: colecistectomia. Interamaricana $1^{\mathrm{a}}$ ed. 1994. p.93-98

17. Wayne H.Schwesinger, MD and Andrew K.Diehl MD - Changing Indications for Laparoscopic Cholecystectomy. Surg Clin N Am. 1996; 76(3):493-501.

18. Laurence Den Besten MD, Jeffrey E, Doty MD. Patogênese e tratamento da coledocolitíase. Clin Cirurg Am Norte. 1981; 917 30

19. Júlio CU, Coellho Alvo O, Vizzoto Jr. Laparoscopic cholecystectomy to treat patients with asymptomatic gallstones. Dig Surg. 2000; 17:344-7
Como citar este artigo:

Gil SM, Braga JF, Centurion SA, Gil BZ. Estudo da incidência de coledocolitíase em pacientes com colecistite calculosa aguda e crônica submetidos à colecistectomia laparoscópica. Rev Col Bras Cir. 2007; 34(4). Disponível em URL: www.scielo.br/rcbc

Endereço para correspondência:

Sidney Moreno Gil

Av. José Nelson Machado, 840 - Parque Iracema

Cep 15800-200 - Catanduva - SP

Email: sgil@skynew.com.br 